antagonist, tocilizumab (TCZ), on NT-proBNP levels and systolic heart function is yet to be obtained.

Objectives: Access the effect of 12 months TCZ therapy on NT-proBNP levels, transthoracal ehocardiography results and analyze the association between congestive heart disease progression and RA activity.

Methods: 37 RA patients (pts) (31F/6M); median age 56,5 [48; 63,5] years; disease duration $48[6 ; 348]$ months; DAS28 score $6,15[5,44 ; 6,45]$; rheumatoid factor (RF)+100\%; anti-citrullinated protein antibody (ACPA) $+79,6 \%$ were treated in an open-label study with TCZ $(8 \mathrm{mg} / \mathrm{kg}$ every 4 weeks). Identification of NT-pro-BNT in blood serum, transthoracal ultrasound evaluation of left ventriculum ejection fraction (LVEF), E/A ratio performed at baseline and 12 months.

Results: $11(29,7 \%)$ pts had congestive heart disease (CHD) (II functional class of NYHA), 7 (18,9\%) pts having signs of mild left ventricular dysfunction (LVD) as dyspnea, shortness of breath, cardiotropic treatment remained the same in the course of the study. After 12 month TCZ treatment as RA activity lowered (DAS28 $2.32[1,75 ; 3,15], p<0,05)$, NT-proBNP levels decreased $(100,95[57.9 ; 117.6] \mathrm{pg} /$ $\mathrm{ml}$ to $90,46[33.62 ; 106.6] \mathrm{pg} / \mathrm{ml})$, along with elevation of $\operatorname{LVEF}(60,75[60 ; 70] \%$ to $67,68[62.5 ; 73.5], p=0,001)$. Increase of $E / A(0,97[0.8 ; 1.17]$ to $1,04[0.7$; 1.42] correlated with decrease of NT-proBNP level $(r=-0,63, p=0,036)$. Raise of LVEF over 12 months correlated with decrease of RA activity according to SDAl scale $(r=-0,670, p<0,05)$. No significant relationship between NT-proBNP levels, LVEF, E/A and other scales measuring RA activity was found. Clinically all patients had improvement in evaluation of their health and no signs of CHD or RVD progression were found.

Conclusion: Use of TCZ in patients with active RA showed none to positive influence on heart condition, specifically, lowering NT-proBNP levels, improving LVEF and reducing clinical signs of LVD.

References:

[1] Pan Y, Li D, Ma J, Shan L, Wei M. NT-proBNP test with improved accuracy for the diagnosis of chronic heart failure. Medicine (Baltimore). 2017 Dec;96(51):e9181.

[2] D Novikova, I Kirillova, E Markelova et al. The first report of significantly improvement of NT-proBNP level in rheumatoid arthritis patients treated with tofacitinib during 12-month follow-up, European Heart Journal, Volume 40, Issue Supplement_1, October 2019, ehz745.0836.

[3] Pappas DA, Nyberg F, Kremer JM et al. Clin Rheumatol. 2018 Sep;37(9):2331-2340.

Disclosure of Interests: None declared

DOI: 10.1136/annrheumdis-2020-eular.5361

\section{AB0309 MEASURING THE DIFFERENCE: COMPARISON OF MEASUREMENT OF FREE INFLIXIMAB ANTI-DRUG ANTIBODIES}

R. Hamilton ${ }^{1}$, S. Shields ${ }^{2}$, A. Mcgucken ${ }^{3,4}$, J. Macdonald ${ }^{2}$, M. Perry ${ }^{3}$, A. Dunlop ${ }^{1}$, E. Gribben ${ }^{5}$, P. Galloway ${ }^{1} .{ }^{1}$ Queen Elizabeth University Hospital, Biochemistry, Glasgow, United Kingdom; ${ }^{2}$ Queen Elizabeth University Hospital, Gastroenterology, Glasgow, United Kingdom; ${ }^{3}$ Royal Alexandra Hospital, Rheumatology, Glasgow, United Kingdom; ${ }^{4}$ University of Glasgow, Infection, Inflammation and Immunity, Glasgow, United Kingdom; ${ }^{5}$ Glasgow Caledonian University, Biochemistry, Glasgow, United Kingdom

Background: Infliximab (IFX) was one of the first TNF alpha inhibitors to be licenced in inflammatory arthritis and is still commonly used today. Studies have shown that approximately $50 \%$ of primary IFX responders will suffer from secondary loss of response within the first 12 months of treatment (1). The development of Anti-Drug Antibodies (ADA's) plays a significant role in this treatment failure (2).

Monitoring of ADA's helps identify those patients who fail to respond to treatment due to low IFX trough levels. In this scenario the presence of ADA's can aid decision-making regarding increasing IFX dosing or switching biologic therapy to optimise treatment. (3).

Objectives: Despite their potential importance the detection of ADAs varies widely depending on the type of assays used. The aim of this study was to determine the qualitative concordance of three commercially available ELISA kits for measurement of free ADAs to IFX on the Grifols Triturus analyser.

Methods: 150 patient samples from patients with inflammatory conditions and low IFX trough drug levels $(\leq 0.6 \mu \mathrm{g} / \mathrm{ml})$ were analysed for free ADAs using Promonitor, Lisa Tracker and IDKmonitor kits on the Grifols Triturus automated ELISA analyser.

Results: Kappa coefficient ( $\mathrm{K}$ ) analysis indicated a moderate agreement between the Promonitor and IDKmonitor assays ( $\mathrm{k}=0.484(95 \% \mathrm{Cl}, 0.357$ to $0.611)$ ) and the IDKmonitor and Lisa Tracker assays ( $\mathrm{k}=0.485(95 \% \mathrm{Cl}, 0.348$ $0.621)$ ) as well as substantial agreement between the Promonitor and Lisa
Tracker assays $(\mathrm{k}=0.768(95 \% \mathrm{Cl}, 0.667-0.870))$. Figure 1 shows the distribution of samples identified as free ADA positive by each kit.

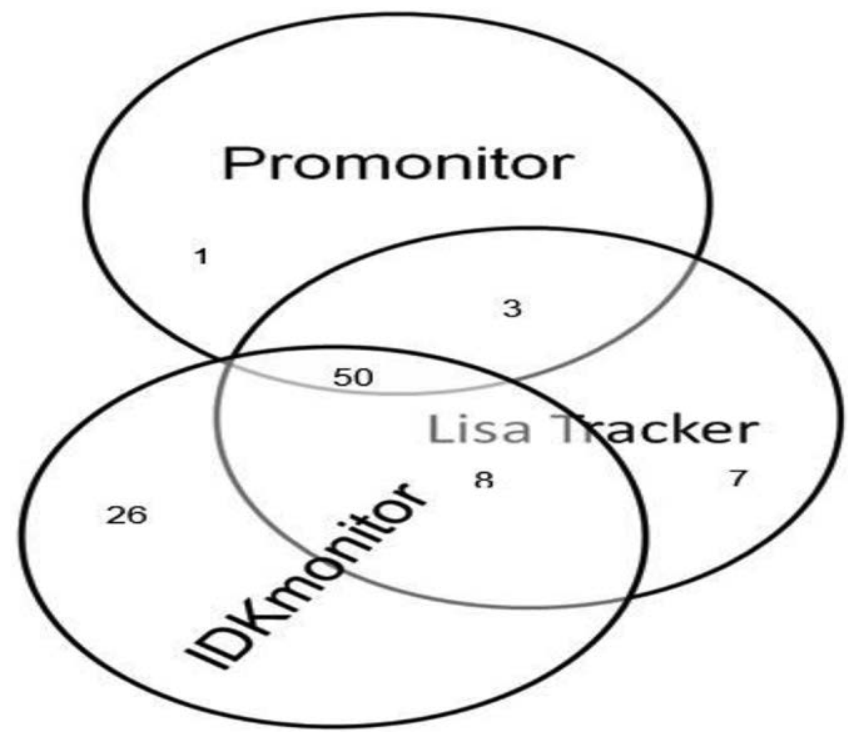

Figure 1. Distribution of samples identified as free ADA positive by Promonitor, Lisa Tracker and IDKmonitor assays. Fifty samples were considered positive by all assays. Three samples were considered positive by Promonitor and Lisa Tracker, and eight samples were considered positive by Lisa Tracker and IDKmonitor. No samples were considered positive by Promonitor and IDKmonitor. One sample was considered positive by Promonitor only, seven samples were considered positive by Lisa Tracker only, and twenty-six samples were considered positive by IDKmonitor

Conclusion: All kits appear amenable for utilisation in a high-throughput laboratory though a true quantitative comparison between these kits was precluded by the absence of any certified reference material for free ADAs to IFX

Although broad qualitative agreement was found between the three kits, they should not be used interchangeably for patient management.

Further research is required to estimate the impact of free ADAs on efficiency of IFX treatment and patient management.

References:

[1] Quistrebert J, Hässler S, Bachelet D et al. Incidence and risk factors for adalimumab and infliximab anti-drug antibodies in rheumatoid arthritis. A European retrospective multicohort analysis. Seminars in Arthritis and Rheumatism Volume 48, Issue 6, June 2019, Pages 967-975 2.

[2] Moots RJ, Xavier RM, Mok CC, Rahman MU, Tsai W-C, Al-Maini MH, et al. (2017) The impact of anti-drug antibodies on drug concentrations and clinical outcomes in rheumatoid arthritis patients treated with adalimumab, etanercept, or infliximab: Results from a multinational, real-world clinical practice, non-interventional study. PLoS ONE 12(4): e0175207. https://doi. org/10.1371/journal.pone.0175207

[3] Smolen JS, Landewé R, Bijlsma J, et al. EULAR recommendations for the management of rheumatoid arthritis with synthetic and biological disease-modifying antirheumatic drugs: 2016 update. Annals of the Rheumatic Diseases 2017;76:960-977.

Disclosure of Interests: Rhona Hamilton: None declared, Stephanie Shields: None declared, Andrew McGucken: None declared, Jonathan MacDonald: None declared, Martin Perry Grant/research support from: Grifols, Abbvie, Sandoz unrestricted educational grant, Consultant of: Abbvie, Gilead, Celltrion Advisory Board, Speakers bureau: Sandoz, Allan Dunlop: None declared, Elaine Gribben: None declared, Peter Galloway: None declared

DOI: 10.1136/annrheumdis-2020-eular.665

\section{AB0310 1 TROUGH CONCENTRATION AND ESTIMATED CLEARANCE CAN DETECT IMMUNOGENICITY TO ADALIMUMAB IN RA PATIENTS: A PROSPECTIVE LONGITUDINAL MULTICENTRE STUDY}

D. Ternant ${ }^{1}$, J. Elhasnaoui ${ }^{2}$, N. Szely ${ }^{3}$, S. Hacein-Bey ${ }^{4}$, A. Gleizes ${ }^{4}$, C. Richez ${ }^{5}$, J. Manson ${ }^{6}$, M. Soubrier ${ }^{7}$, O. Brocq ${ }^{8}$, J. Avouac ${ }^{9}$, A. FogdellHahn $^{10}$, P. Dönnes ${ }^{11}$, G. Paintaud ${ }^{1}$, C. Desvignes ${ }^{1}$, F. Deisenhammer ${ }^{12}$, S. Spindeldreher ${ }^{13}$, M. Pallardy ${ }^{14}$, X. Mariette ${ }^{15}$, D. Mulleman ${ }^{16}$ on behalf of the ABIRISK [Anti-Biopharmaceutical (BP) ImmunizationPrediction and Clinical Relevance to Reduce the Risk] European consortium. 